\title{
Diagnóstico histopatológico de entamoebiosis en un primate en cautiverio (Alouatta caraya)
}

\author{
Montenegro, M.; Sánchez Negrette, M.; Lértora, W.J. \\ Cátedra de Patología General y Sistemática, Facultad de Ciencias Veterinarias, Universidad Nacional del \\ Nordeste, UNNE, Sargento Cabral 2139, Corrientes (3400). Tel. 03783-425753. \\ E-mail: patgral@vet.unne.edu.ar.
}

\begin{abstract}
Resumen
Montenegro, M.; Sánchez Negrette, M.; Lértora, W.J.: Diagnóstico histopatológico de entamoebiosis en un primate en cautiverio (Alouatta caraya). Rev. vet. 21: 1, 59-62, 2010. La entamoebiosis es una enfermedad causada por Entamoeba histolytica, protozoario que puede vivir en el intestino grueso sin causar daños y que se disemina mediante el agua o alimentos contaminados con heces del portador. El hombre y algunos primates no humanos son los huéspedes naturales. El objetivo del presente trabajo fue comunicar la ocurrencia de entamoebiosis en un mono carayá macho (Alouatta caraya), que ingresó al zoológico de la ciudad de Corrientes (Argentina) cuando tenía 2 meses de edad, siendo alimentado con mamadera en un recinto compartido con otros tres monos de su misma edad. Luego de su muerte y realizada la necropsia, todos los órganos fueron fijados en formol al $10 \%$ y procesados por las técnicas histológicas clásicas para bloques parafinados. Los protozoarios fueron observados en mucosa, submucosa y luz intestinal, como estructuras ameboides en diferentes estadios de evolución, formando galerías a todo lo largo de la mucosa. La submucosa presentó edema e infiltrado mononuclear. La presencia de esta parasitosis en un primate de temprana edad, induce a reflexionar sobre la importancia del manejo en cautiverio, donde la conservación exitosa de los ejemplares en resguardo dependerá de múltiples factores que deberían ser tenidos en cuenta al momento de diseñar programas de contención y traslado de animales silvestres.
\end{abstract}

Palabras clave: Alouatta caraya, Entamoeba histolytica, lesiones histopatológicas.

\begin{abstract}
Montenegro, M.; Sánchez Negrette, M.; Lértora, W.J.: Histopathological diagnosis of entamoebiosis in a captive primate (Alouatta caraya). Rev. vet. 21: 1, 59-62, 2010. Entamoebiosis is a disease caused by Entamoeba histolytica, a protozoan that can live in the large intestine without causing disease and spreads through water or food contaminated with feces from being humans and other primates, the natural hosts. The aim of this study was to report the occurrence of entamoebiosis in a 2 months-old male howler monkey (Alouatta caraya), located at the zoo of Corrientes City (Argentina). Animal was bottle-fed artificially and housed with three monkeys of the same age. After natural death, necropsy was performed and organs were fixed in $10 \%$ formalin and processed by conventional histological techniques for paraffin blocks. Protozoa were observed throughout the mucosa and submucosa and in the intestinal lumen as amoeboid structures at different stages of evolution, forming galleries throughout the mucosa. The submucosa showed edema and mononuclear infiltration. The presence of this parasite in a young primate emphasises the importance of captivity management, where the successful conservation of species depend on multiple factors that should be taken into account when designing management programs.
\end{abstract}

Key words: Alouatta caraya, Entamoeba histolytica, histopathological findings.

\section{INTRODUCCIÓN}

La entamoebiosis es una enfermedad causada por Entamoeba histolytica, protozoario que puede vivir en el intestino grueso sin causar daño, aunque en ocasiones invade las paredes del colon provocando colitis, disentería aguda o diarrea prolongada en los casos crónicos

Recibido: 2 mayo 2010 / Aceptado: 22 junio 2010
9,11, 12. El hombre y algunos primates no humanos son los huéspedes naturales. Sin embargo, algunos mamíferos como el perro y el gato pueden también albergar E. histolytica. Este protozoario se disemina a través del agua o alimentos contaminados con heces del portador, propagándose a través de las manos, alimentos contaminados, moscas y cucarachas, entre otros ${ }^{3,11}$.

El ciclo del parasito consta de una forma infectante quística de alrededor de 10 a $15 \mu \mathrm{m}$ con una pared de 
quitina que se desarrolla fuera del huésped. La forma no infectante se halla dentro del huesped y en las heces frescas ${ }^{2}$. El ciclo vital de E. histolytica depende de la excreción de los quistes por las heces y de la subsiguiente ingestión en otro huésped ${ }^{9,12}$. La forma quística infectante presenta una pared de quitina y cuatro núcleos, siendo resistente a la acidez gástrica. Una vez ingeridos, los quistes colonizan la superficie de células epiteliales secretoras de mucina del colon y allí liberan trofozoitos, formas ameboides que se reproducen bajo condiciones anaerobias sin dañar al huésped ${ }^{12}$.

La patogenicidad es influenciada por la dieta, el estado inmunitario del huésped y la virulencia atribuida a los diferentes estadios del parásito. En los tejidos, $E$. histolytica semeja a un macrófago por el tamaño y el gran número de vacuolas dentro de su citoplasma ${ }^{2}$. Cuando se adhiere al epitelio del colon produce disentería debido a la presencia de lectinas capaces de efectuar degradación enzimática del mucus y adherencia al epitelio del huésped, mecanismo importante para la producción del daño tisular. Una vez producida la invasión del epitelio de las criptas, penetran en la mucosa y submucosa provocando una reacción neutrofílica ${ }^{12}$.

Limitados estudios han documentado las parasitosis intestinales de poblaciones de primates, tratándose en su mayoría de animales de cautiverio ${ }^{4,8,10}$. Entre
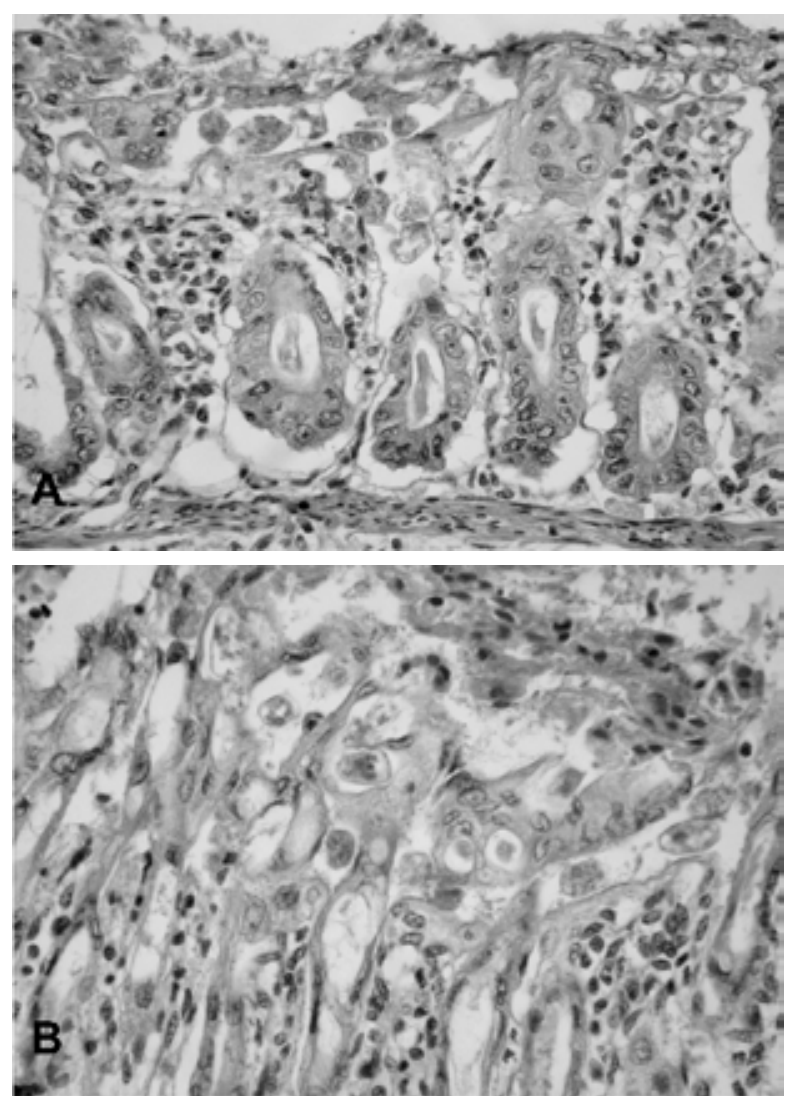

Figura 1. A: mucosa intestinal con numerosas formas parasitarias ameboides, infiltrado inflamatorio monomorfonuclear de la lámina propia y necrosis $(\mathrm{HyE}$, 20X). B: las mismas lesiones, a mayor aumento (HyE, 40X). ellos, el género Alouatta es uno de los más estudiados respecto a sus parasitosis intestinales $5,7,13,15,16$.

El objetivo del presente trabajo fue comunicar la ocurrencia de entamoebiosis en un mono carayá (Alouatta caraya) en cautiverio, así como caracterizar las lesiones desde el punto de vista histopatológico.

\section{PRESENTACIÓN DEL CASO}

La entamoebiosis se presentó en un mono carayá macho (Alouatta caraya), que había ingresado al zoológico de la ciudad de Corrientes (Argentina) con aproximadamente 2 meses de edad. Fue alimentado con mamadera con una mezcla de leche deslactosada, banana y manzana durante dos meses y medio, en este tiempo compartió el hábitat con otros tres monos de su misma especie y edad. Luego de este período fue separado del grupo y alojado en una jaula compartida con una hembra adulta, con alimentos sólidos. La muerte del animal fue precedida por un período de dos semanas de inapetencia y decaimiento, evidenciando mucosas pálidas y alopecia en cabeza y pies. Fue tratado con antibióticos y fluidoterapia sin resultados satisfactorios. Realizada la

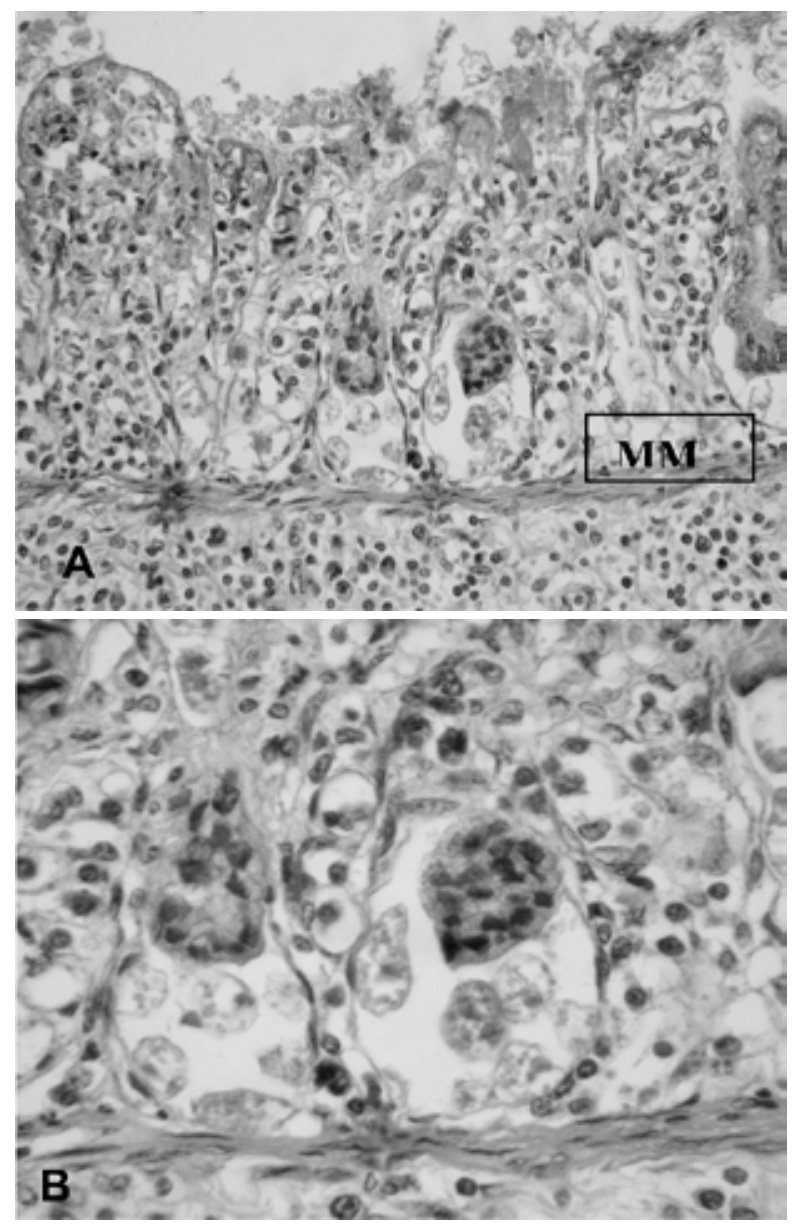

Figura 2. A: numerosos parásitos en la mucosa intestinal, en contacto con la muscular de la mucosa "MM" (HyE, 20X). B: formas ameboides con citoplasma espumoso y núcleos centrales característicos en glándulas de Lieberkuhn (HyE, 40X). 
necropsia, todos los órganos fueron fijados en formol al $10 \%$, procesados por las técnicas histológicas clásicas para bloques parafinados, cortados en micrótomo en 5 $\mu \mathrm{m}$ y coloreados con hematoxilina y eosina (HyE).

\section{RESULTADOS}

El estudio histopatológico del intestino, principalmente del colon, reveló necrosis y erosión en todo el espesor de la mucosa, con edema de la submucosa e infiltrado inflamatorio de tipo monomorfonuclear que comprometió la mucosa y submucosa, compuesto principalmente por linfocitos, plasmocitos y macrófagos.

Los parásitos fueron observados en la mucosa y submucosa como estructuras esféricas irregulares, de diferentes tamaños y citoplasma espumoso con núcleos centrales, muy similares a macrófagos (Figura 1). En la mucosa, las formas ameboides (trofozoitos) se hallaron aisladas o agrupadas, en su mayoría dentro de las glándulas de Lieberkuhn (Figura 2); los parásitos también fueron hallados en la submucosa y dentro de vasos sanguíneos.

En todos los cortes estudiados fue llamativa la formación de galerías o canales a lo largo de la mucosa, dispuestos en forma paralela a la superficie, contenien-

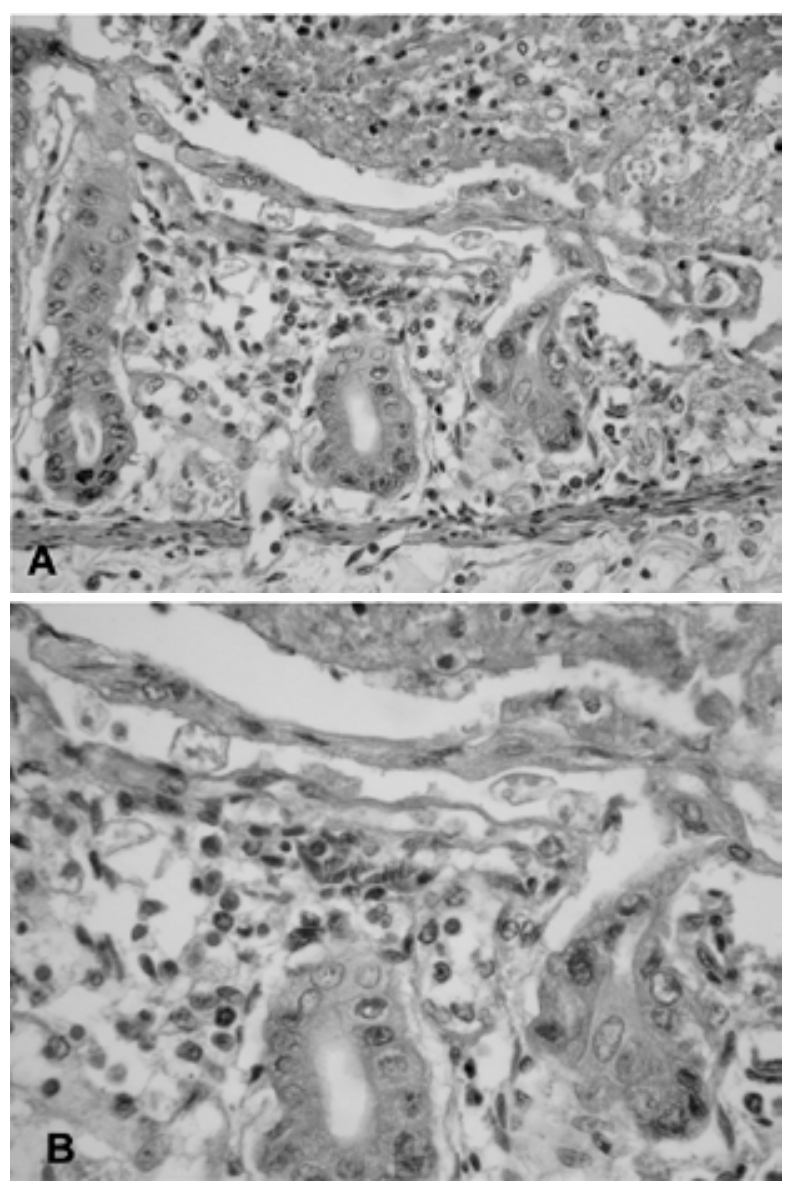

Figura 3. A: formación de galerías a lo largo de la mucosa, infiltrado inflamatorio y necrosis en superficie. B: galerías con varias formas parasitarias en su interior (HyE, 40X). do numerosas formas ameboides (Figura 3). La luz intestinal también presentó gran cantidad de parásitos en diferentes estadios de evolución, formas quísticas y otras carentes de la cubierta de quitina formando racimos de cuatro núcleos junto a restos alimenticios, detritus celulares y mucus.

\section{DISCUSIÓN}

La relación parásito-huésped ha actuado siempre como una importante fuerza de selección, la cual ha afectado la densidad y distribución de las especies ${ }^{1}$. En este sentido la infección por endoparásitos es común en los primates no humanos y la mayor parte de los estudios que se han realizado al respecto han estado restringidos a animales en cautiverio ${ }^{4,8}$. Los primates son particularmente vulnerables a los efectos de las infecciones por protozoarios, debido a que generalmente viven en grupos sociales cerrados que facilitan la transmisión ${ }^{5,13}$.

Entre los principales factores que influyen en el parasitismo y en las infecciones gastrointestinales de primates silvestres se citan cambios en la densidad, comportamiento, edad, condición reproductiva y dieta, así como la humedad y la fragmentación del hábitat. La dieta y el estado inmunitario del huésped, sumado a la virulencia atribuida a los diferentes estadios del parásito influencian su patogenicidad ${ }^{6,7,14}$. Los primates que exhiben un mayor comportamiento social son más susceptibles a infectarse que los que tienen menos contacto con otros individuos. Este contacto social está determinado por la especie del primate, edad, género y rango social del individuo ${ }^{5,13,17}$. En el presente caso, varios son los factores que pudieron haber influenciado la aparición de esta enfermedad, tales como la edad del primate, de apenas 2 meses, aislado de sus padres e introducido a un ambiente sucedáneo, bajo alimentación artificial.

Desde el punto de vista del diagnóstico histopatológico, la entamoebiosis aguda (como la del presente caso) produce una colitis necrótica-ulcerativa de fácil diagnóstico, principalmente cuando los protozoarios aparecen en diferentes estadios y también cuando se evidencian lesiones características propias de esta parasitosis. Las lesiones iniciales consisten en erosiones de la superficie epitelial en su parte profunda y úlceras. Los parásitos pueden hallarse en el mucus de la superficie del colon, también pueden ser visibles en mayor cantidad en el exudado sobre las erosiones o sobre las lesiones ulceradas superficiales. Las úlceras y las áreas de necrosis presentan infiltrado predominantemente neutrofílico y la pérdida de las estructuras glandulares puede extenderse a casi toda la mucosa. Conforme progresa la lesión, los parásitos cavan en sentido lateral formando galerías y la mucosa superficial subyacente sufre necrosis y es desprendida ${ }^{2}$.

En los tejidos, E. histolytica a menudo está rodeada por un halo claro, adoptando formas esféricas o irregulares, con extensiones de seudópodos cuyos diámetros 
oscilan entre 6 y $50 \mu \mathrm{m}$. En el centro de los núcleos existe un denso cariosoma y la cromatina periférica es grumosa. El citoplasma, generalmente espumoso, puede albergar restos de eritrocitos en el fagolisosoma, glicógeno positivo con la coloración PAS.

La presencia de esta parasitosis en un primate de temprana edad, induce a reflexionar sobre la importancia del manejo de especies silvestres en cautiverio. La conclusión emergente es que la conservación exitosa de los ejemplares en resguardo dependerá de factores como densidad de animales, edad, condición reproductiva, dieta, temperatura y humedad de los recintos, amén de otras condiciones del hábitat. Tales requerimientos deberían ser tenidos en cuenta al momento de diseñar programas de manejo y traslado de primates, para minimizar los efectos deletéreos de las infecciones parasitarias.

\section{REFERENCIAS}

1. Anderson RM. 1979. Parasite pathogenecity and the depression of host population equilibria. Nature 279: 150-152.

2. Brown CC, Baker DC, Barker IK. 2007. Alimentary system. In: Jubb, Kennedy \& Palmers: Pathology of Domestic Animals (Maxie MG Ed), $5^{\text {th }}$ ed, Vol 2, Saunders, Philadelphia, p. 277.

3. Campillo MC, Vázquez FA, Fernández AR, Acedo MC, Rodríguez SH, López-Cozar IN, Baños PD, Romero HQ, Varela MC. 1999. Parasitología Veterinaria, McGraw-Hill, Madrid, p.968.

4. Fuentes R. 1986. Incidencia de amebiasis en los primates del zoológico de San Juan de Aragón. Tesis FMVZ, Universidad Nacional Autónoma de México, DF, p.128.

5. Freeland WJ. 1983. Parasites and the coexistence of animal host species. Am Nat 2: 223-236.

6. Gillespie TR, Greiner EC, Chapman CA. 2004. Gastrointestinal parasites of the guenons of Western Uganda. $J$ Parasitol 90: 1356-1360.
7. Gillespie TR, Chapman CA, Greiner EC. 2005. Effects of logging on gastrointestinal parasite infections and infection risk in African primates. $J$ Appl Ecol 42: 699-707.

8. Gual F, Guerrero C, Quiroz H. 1990. Determinación de parásitos gastroentéricos en primates del Zoológico de Chapultepec. Tesis FMVZ, Universidad Nacional Autónoma de México, DF, p. 146.

9. Haque R, Huston C, Hughes M, Houpt E, Petri WA. 2003. Amebiasis. N Eng J Med 348: 1565-1573.

10. Jessee MT, Schilling PW, Stunkard JA. 1970. Identification of intestinal helminth eggs in old world primates. $\mathrm{Lab}$ Anim Care 20:83-87.

11. McGavin DM, Zachary JF. 2007. Pathologic basis of veterinary disease, $4^{\text {th }}$ ed., Mosby, St.Louis (USA), p. 384.

12. Kumar V, Abbas AK, Fausto N. 2005. Robbins \& CotranPatología estructural y funcional, $7^{\mathrm{a}}$ Ed, Elsevier, Madrid, p.843-844.

13. Stoner KE. 1995. Prevalence and intensity of intestinal parasites in mantled howling monkeys (Alouatta palliata) in northeastern Costa Rica: implications for conservation biology. Conservat Biol 2: 539-546.

14. Stoner KE, González AM, Maldonado S. 2005. Intestinal parasite infections in primates: implications for conservation. Rev Universidad \& Ciencia (Tabasco, México), 2: 61-72.

15. Santa Cruz A, Borda J, Patiño E, Gomes L, Zunino G. 2000. Habitat fragments and parasitism in howler monkeys (Alouatta caraya). Neotrop Prim 8: 146-148.

16. Serrano MA. 1998. Incidencia de protozoarios gastrointestinales en primates del Zoológico Zacango de Calimaya, Estado de México. Tesis FMVZ, Universidad Autónoma del Estado de México, Toluca, p. 98.

17. Wolff PL. 1993. Parasites of new world primates. In: Zoo and wild animal medicine (Fowler ME Ed), Saunders, Philadelphia, p. 644. 